\title{
The Electronic Computer A Machine with a Past
}

\section{G. A. Erskine}

It is about 130 years since the wealthy mathematician and amateur engineer, Charles Babbage, conceived the idea of a completely automatic calculating machine.

Babbage realized that if his machine was to operate without human intervention, it would have to print its results and would require an internal store or 'memory' in which intermediate results of the calculation could be placed for subsequent use by the machine. This store was to consist of a thousand columns of digit wheels, each column containing 50 decimal digits. The automatic contro! of the sequence of arithmetic operations was to be provided by perforated cards of the kind used for controlling the lifting of the warp threads in a Jaquard loom.

With remarkable insight, Babbage realized that a truly universal calculating machine would need to vary its sequence of operations depending on the unforeseeable result of intermediate calculations. He therefore provided for a mechanism that would cause the machine to skip forwards or backwards over a specified number of control cards when the number contained on some specified storage column changed its sign. He also provided for the automatic detection of the overflow which would occur if any result exceeded 50 digits in length, and he foresaw the use of what would now be called programmed multilength arithmetic to deal with such cases. Thus Babbage anticipated many of the essential features of the modern computer.

Babbage prepared detailed drawings of many parts of his Analytical Engine and spent much of the remainder of his life experimenting with pieces of the mechanism. During a visit to Italy, he described the proposed machine to a small group of people which included L.F. Menabrea, an army engineer and mathematician. Menabrea was so much interested by Babbage's machine that he published $a$ general description of it in the Bibliothèque Universelle de Genève in 1842.

In the same year, Lady Augusta Lovelace, daughter of the poet Byron, published an English translation of Menabrea's article, to which she added explanatory appendices which were three times the length of the original article. Lady Lovelace, mathematician and a friend of Babbage, understood clearly what was involving in program- ming' the Analytical Engine, possibly more clearly than Babbage himself. One of her appendices gives a complete - and completely modern - program for calculating the successive Bernoulli numbers by means of a recurrence relation (see page 165). The program shows how the repetitive nature of the calculation was to be exploited by the machine.

Since even Babbage's substantial fortune was not sufficient to pay for the cost of constructing the Analytical Engine, Babbage and Lady Lovelace devised, and put into practice, an infallible system for backing horses. The results were disastrous.

\section{The Twentieth Century}

From the time of Babbage's death in 1871 until the late 1930s, there seems to have been no attempt to construct a completely automatic general-purpose calculator. Manually operated calculators underwent a steady. improvement and electromechanical punched card machinery was invented and developed. Such machines could sort, add, subtract, and print, but there was no means of specifying that an arbitrary sequence of arithmetic operations should be carried out without manual intervention.

In 1939, Howard Aiken of the University of Harvard entered into collaboration with the International Business Machines Corporation to construct an automatic electro-mechanical calculator in which the sequence of operations was to be controlled by a perforated paper tape, in rather the same way as Babbage's machine (of which Ajken knew nothing at the time) was to be controlled by Jaquard cards. This machine, the Harvard Mark 1 calculator, was completed in 1944.

In the meantime, work had begun at the University of Pennsylvania on the first completely electronic computer, the ENIAC This computer, designed by J.W. Mauch!y and J. P. Eckert under a contract with the United States Army, was completed in 1946. Vacuum tube circuits were used to provide arithmetic capabilities together with internal storage for twenty 10-digit numbers. Each 10-digit storage location required 550 tubes, and the whole computer used approximately twenty thousand tubes. Programming was by means of switches and plug-in wires, and the time required to set up a new calculation might be anything from a few hours to several days. The ENIAC was certainly fast: addition took 200 mircoseconds $(200 \mu \mathrm{s})$ and multiplication 3 milliseconds ( $3 \mathrm{~ms}$ ).

Some time in the summer of 1944, while the ENIAC was still under construction, the mathematician John von Neumann learnt of the ENIAC in an accidental meeting with a colleague, H.H. Goldstine. It is tempting to think of von Neumann as being horrified by the thought of those twenty thousand vacuum tubes being used to provide such inflexible computing facilities. In any case, von Neumann and Goldstine, in collaboration with members of the ENIAC team, set to work on the logical principles of an electronic computer which would be far more versatile than the ENIAC while using much less hardware. These principles were presented to an international audience at a summer school held at the University of Pennsylvania in 1946, and were subsequently published in a series of reports.

The von Neumann Computer

The computer proposed by von Neumann and Goldstine in 1946 is essentially the modern computer. The fundamental proposal was that the 'instructions', which specify the sequence of operations to be performed, should be coded into numerical form and held in the same high-speed storage device as the numbers on which the computer was to operate. The computer could then extract successive instructions from the store at the same speed as it could extract numbers, and could, when necessary, be made to jump easily from one place to another in the instruction sequence according to the result of some simple test (for example, a test on the sign of the number in a specified storage location). Using an input medium such as paper tape, it would be possible to load in a few seconds a program containing hundreds of instructions, thus preparing the computer for a new calculation.

The von Neumann and Goldstine reports also describe the use of a library of programs (already foreseen by Babbage) and the means by which sub-routines (sequences of instructions for performing 
The first programmer, Ada Augusta, the Countess of Lovelace, and (opposite) the first programm, prepared by Lady Lovelace in 1842 for calculating the Bernoulli numbers on Babbage's Analytical Engine.

(Reproduced from Lord Bowden's book

'Faster Than Thought' with acknowledgement to Lady Wentworth). frequently occurring operations), prepared in a form which was independent of their final position in the store, could be 'relocated' before the beginning of a calculation.

One effect of the 1946 summer school was to start a computer-building race. This race was won by a team headed by M.V. Wilkes of the University of Cambridge, whose EDSAC computer came into operation in May 1949. Only shortly behind came the University of Manchester with its computer incorporating the first index registers.

The Vanishing Programmer

The designers of the early computers assumed that programming would be in the hands of a small group of specialists, probably mathematicians, and that it would be undesirable to make the task too easy. For example, von Neumann and Goldstine presented the following argument against providing built-in floating-point arithmetic: 'The floating binary point represents an effort to render a thorough mathematical understanding of at least part of the problem unnecessary, and we feel that this is a step in a doubtful direction'. Thinking along the same lines, the University of Manchester adopted for their first computer a programming code in which all instructions were constructed from a group of 32 teleprinter characters. Thus the sequence of instructions for calculating a sum of the form $a_{1} b_{1}+a_{2} b_{2} \ldots$ $+a_{16} b_{16}$ became

$$
\begin{aligned}
& \text { VEQO } \\
& / \text { I UK } \\
& / \text { UUF } \\
& \text { \&EQG } \\
& \text { E / / T }
\end{aligned}
$$

This programming code was retained when engineered versions of the Manchester University computer were offered for sale commercially! Gradually, however, the manufacturers of computers began to wonder whether perhaps difficulties of programming might not be discouraging prospective customers. This led to the development of simplified programming languages, of which Fortran (introduced in 1957) has been one of the most successful. The sequence of instructions given

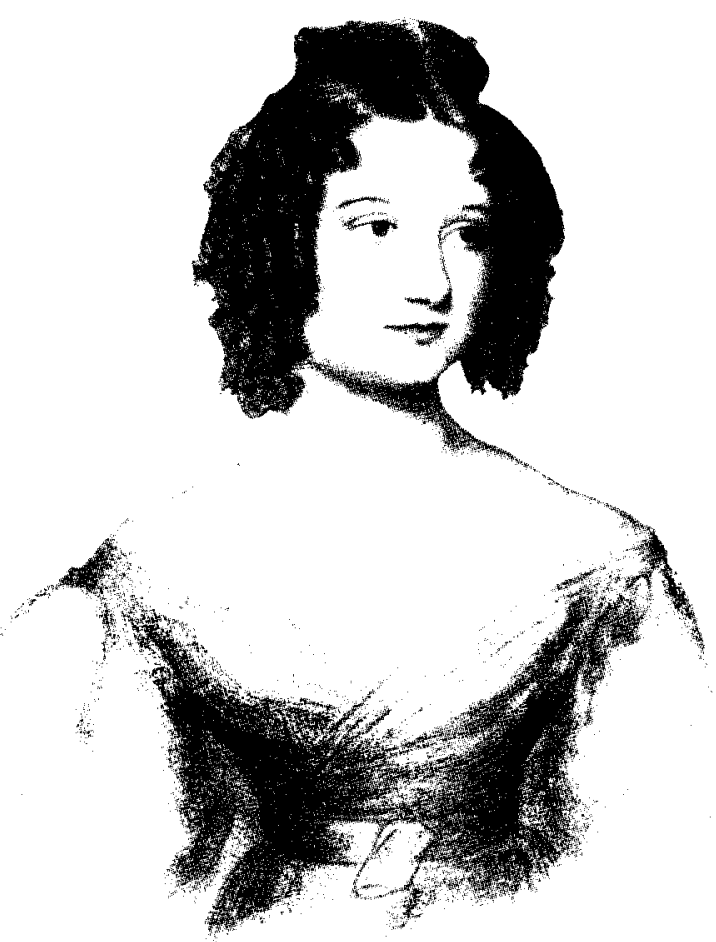

above can be written in Fortran as follows: SUM $=0.0$ DO $10 \mathrm{~J}=1,16$

10 SUM $=$ SUM $+A(J){ }^{*} B(J)$

where the asterisk denotes multiplication. In this way, programming became a doit-yourself' activity, and the only full-time programmers to remain in business were those whose job it was (and is) to write the complicated compilers and supervisory programs which constitute the vital' behindthe-scenes 'software' of the modern computer.

\section{The Communication Gap}

It was soon realized that the electronic computer, invented to do arithmetic quickly, could also perform non-numerical tasks. Computers can be programmed to play a good game of draughts, but only an indifferent game of chess. They can make poor translations from one language into another. If connected to suitable equipment, they can read printed text or control machine tools. The only limit to their capabilities, apart from their finite - but ever-increasing - speed and storage capacity, seems to lie in the intellectual limitations of those who programme them.

Since a computer cannot execute everyone's program at once, programs and data (in the form of punched cards or perforated paper tape) are usually placed in a queue in which they wait until they can be processed. The results generated by programs may also queue on some largecapacity 'backing-store' until they can be printed. The delay introduced by these queues constitutes the 'turn-around-time', often amounting to several hours.

For many computer applications, this delay is not acceptable. For example, computers have been successfully programmed to do algebra and simple calculus. But who wants to do algebra by computer if he must wait several hours between making a minor change to a formula and receiving a printed result?

In principle, the answer to this objection lies in the introduction of a time-sharing console system similar to the MAC project of the Massachusetts Institute of Technology. The user of such a system sits at an electric typewriter which is connected to a large computer and types in programs or data and receives back results, ignoring the fact that the computer is simultaneously providing a similar service to other typewriters. In fact, the computer is rapidly servicing the users in sequence, sharing its time equitably between them. Unfortunately, if there are too many users. the delay between typing a request and receiving a reply becomes intolerably long; and no computer at present in existence could provide an adequate service to all of the 250 or so people who use the main CERN computer each day.

Perhaps there never will be computers big enough and fast enough to provide this kind of on-line service to all who want to use them. If small computers continue to become simultaneously faster and cheaper, the use of a big computer may become the exception rather than the rule. But whatever the direction of the future development, we shall presumably see a continuing increase in Babbage's hoped-for 'substitution of machinery, not merely for the skill of the human hand, but for the relief of the human intellect'. What will happen when the human hand and the human intellect have been sufficiently relieved remains to be seen. 


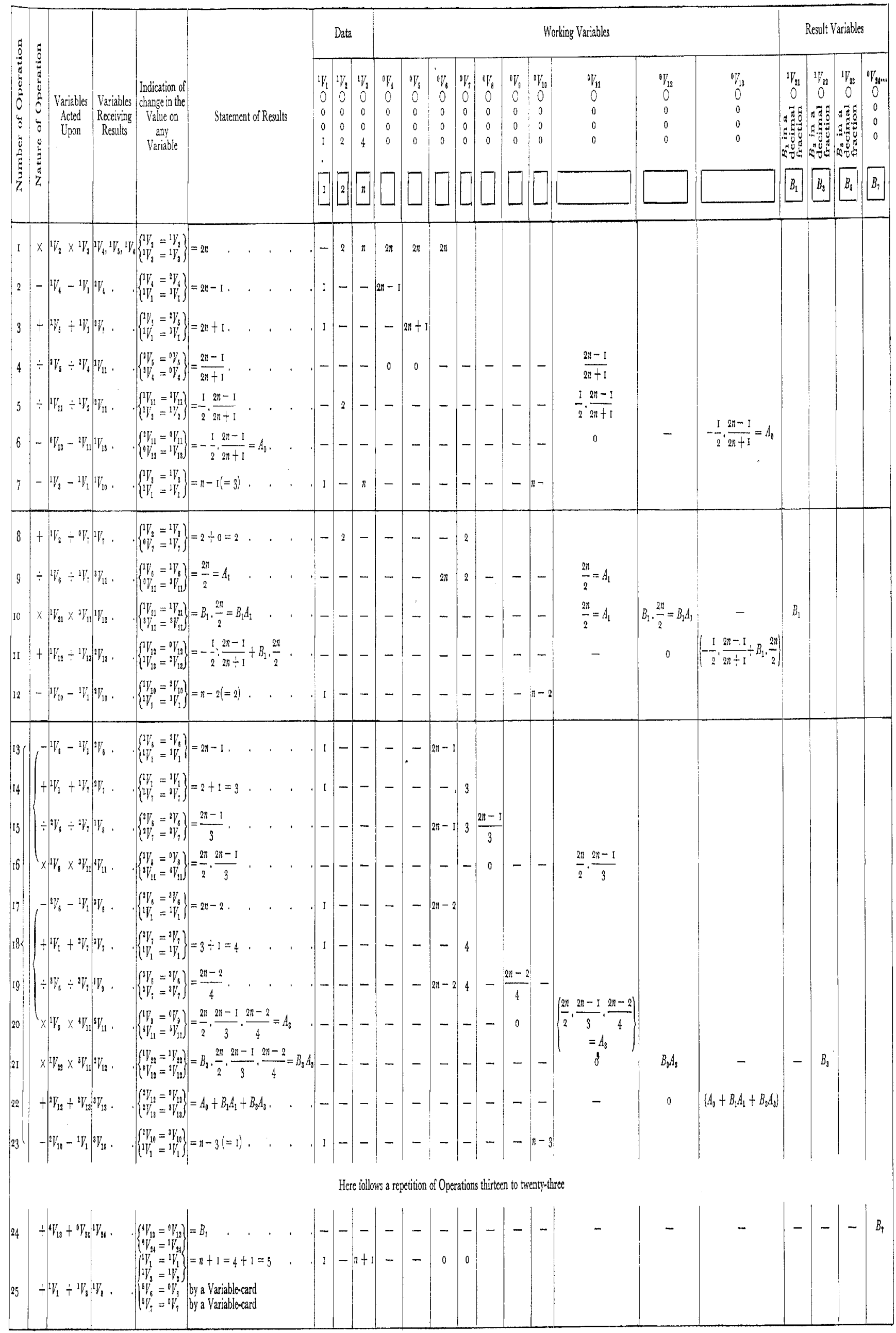

